\title{
Mujeres jóvenes y ciudadanía en Chile: Una mirada a las nuevas configuraciones políticas
}

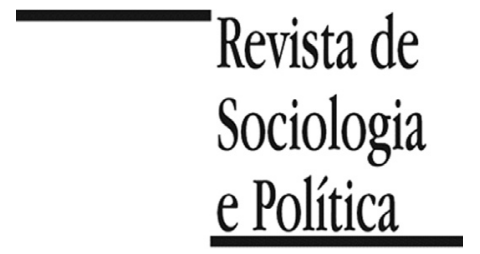

DOI 10.1590/1678-987316245902

\author{
Bruno Bivort, Soledad Martínez-Labrín, \\ Cristian Orellana y Fernando Farías
}

\begin{abstract}
Resumen
En el marco de los cambios producidos en las formas en que los y las jóvenes se vinculan en su calidad de ciudadanos/as con el Estado, el presente trabajo tiene como propósito identificar y analizar las dimensiones distintivas que pudieran otorgar una mayor profundidad y amplitud a la categoría ciudadanía, desde la perspectiva de las mujeres jóvenes, visibilizando las particularidades asociadas a su doble condición, de jóvenes y de mujeres. Utilizando una metodología integrada, en este estudio exploratorio se Ilevó a cabo un procesamiento de 156 respuestas de mujeres jóvenes a un cuestionario on-line sobre ciudadanía y género. A partir de ello, se evidencia la visión de los derechos que las jóvenes priorizan como ciudadanas y mujeres, los temas específicos que consideran necesarios de impulsar desde una agenda ciudadana y se pone especial énfasis en las situaciones o ámbitos percibidos como espacios de discriminación de género para las mujeres jóvenes, y que habitualmente, quedan inadvertidos en estudios sobre ciudadanía, que no incorporan la dimensión de género en su análisis. Esto permite dar cuenta de una mayor complejidad conceptual para mirar la ciudadanía, más allá de las concepciones tradicionales.
\end{abstract}

PALABRAS-CLAVE: género; ciudadanía; mujeres jóvenes; discriminación; participación política.

Recibido em 22 de Diciembre de 2014. Aceptado em 16 de Mayo de 2015.

\section{Introducción ${ }^{1}$}

\footnotetext{
1 Este artículo es producto del Proyecto de Investigación sobre Representaciones Sociales de Ciudadanía desde una perspectiva de Género Código 154224 4/R y del trabajo del Grupo de Investigación en Género, Ciudadanía y Equidad Código 122024 GI/EF de la Dirección de Investigación de la Universidad del Bío-Bío. Agradecemos las sugerencias y comentarios de los dictaminadores anónimos de la Revista de Sociologia e Política.
}

$\mathrm{E}$ presente trabajo tiene como propósito reflexionar sobre las diferencias vinculadas a la condición de género y que se encuentran presentes en las demandas ciudadanas, desde la perspectiva de las mujeres jóvenes. En dichas demandas se evidencia que existen particularidades y que es posible distinguir dimensiones que otorgan mayor profundidad y amplitud al concepto de ciudadanía, emergiendo categorías como el cuerpo, lo laboral, la discriminación y la violencia simbólica, entre otras, que aparecen fuertemente asociadas a lo político e institucional.

Se analiza la visión que las mujeres jóvenes poseen sobre sus derechos en tanto ciudadanas, y las dimensiones diferenciales que establecen respecto de los derechos ciudadanos generales, así como los temas específicos que consideran prioritarios de impulsar desde una agenda ciudadana. Adicionalmente, se pone especial énfasis en situaciones o ámbitos percibidos como espacios de discriminación de género por las mujeres, y que habitualmente, quedan inadvertidos en estudios sobre ciudadanía que no incorporan la dimensión de género en su análisis, lo que permite dar cuenta de una mayor complejidad conceptual para mirar la ciudadanía, más allá de las concepciones tradicionales.

La preocupación por la situación de los/las jóvenes respecto de la participación ciudadana, está dada por la importancia estratégica que tiene el desempeño actual y futuro de este grupo etario en el devenir de los países, y por que la mayoría de los estudios realizados dan cuenta de un proceso de distanciamiento de este sector de la población respecto de las instituciones democráticas 
tradicionales, lo que permite evidenciar un conjunto de prácticas entre los/las jóvenes, que se ubican al margen de la participación en las instituciones formales de la política (Bonvillani et al., 2008; Rodríguez 2010; Zarzuri 2010).

Además, y en relación con lo anterior, se ha optado por el grupo juvenil debido al contexto político que ha predominado en Chile en los últimos años, caracterizado por un fuerte rechazo a la institucionalidad vigente, y a las prácticas políticas tradicionales, evidenciado en altos porcentajes de abstención en los procesos eleccionarios, lo que ha llegado a poner en jaque la legitimidad del orden imperante. El distanciamiento juvenil con las instituciones políticas que para muchos/as es señal de apatía, para otros/as no es sino la lectura en el sentido opuesto de un fenómeno que está llevando a los/las jóvenes a pensar y actuar políticamente de formas no convencionales, donde el voto puede o no ser un instrumento de acción política, pero de todos modos, pareciera no ser el único (Aguirre \& Agustín 2015). Estamos aquí frente a un cambio cultural, en el contexto de una democracia representativa, que limita o excluye a la ciudadanía y pone el énfasis en políticas públicas de carácter tecnocrático, contribuyendo al debilitamiento de la acción ciudadana (Paredes 2011). En esta misma dirección, para Toro (2008) la mayor o menor afección juvenil con los procesos políticos tradicionales se relaciona de forma directa con los vínculos que las instituciones establecen con la ciudadanía; es decir, que a mayor confianza de los/las jóvenes en las organizaciones políticas y de gobierno, la probabilidad de ser parte del proceso electoral aumenta.

Sumado a ello, se evidencia una ausencia histórica de estudios sobre las mujeres en asuntos de ciudadanía (Giordano 2012), por lo que resulta relevante visibilizar las nuevas formas en que las y los jóvenes en general se aproximan a lo político (Aquin 2003; Bermúdez, Savino \& Zenklussen 2004; Sandoval \& Hatibovic 2010; Sandoval 2012), pero especialmente, cómo lo están haciendo las jóvenes.

Desde esta perspectiva, conocer las opiniones que las jóvenes en tanto actoras políticas activas o pasivas, poseen sobre sus derechos como ciudadanas, sus propias prioridades, y los ámbitos de discriminación, identificados desde una perspectiva de género, podría contribuir a una mejor comprensión de un fenómeno, que ha sido habitualmente leído en clave adultocéntrica y masculina, por lo que se espera que los resultados sirvan para contar con una visión más cercana al mundo de las mujeres jóvenes, ya que la forma en que ellas representan su participación ciudadana, incide en la forma en cómo la ejercen y constituyen prácticas que están configurando un nuevo escenario social.

\section{Marco referencial}

En esta investigación, se parte de la base de que la ciudadanía es un concepto complejo, cuya permanente construcción se desarrolla en la relación entre las Personas, El Estado y La Sociedad, y que asume particularidades contextuales e históricas, abarcando en la actualidad distintas dimensiones, que se extienden a diversos ámbitos de la vida. Para comprender el actual concepto de ciudadanía, entonces, es necesaria la inclusión de aspectos subjetivos que pueden estar vinculados a las expectativas y/o a la autopercepción, así como el análisis de elementos macro-sociales (Cortina 1997; Marshall \& Bottomore 1998; Gutiérrez 2011).

La noción de ciudadanía, sin embargo, ha transitado por varios momentos conceptuales. Su definición tradicional, desde quienes han sido precursores clásicos de su conceptualización desde mediados del siglo XX, habla de que "La ciudadanía es una condición otorgada a aquellos que son miembros plenos de una comunidad. Todos los que poseen la condición son iguales con respecto 
a los derechos y deberes de que esta dotada esa condición" (Marshall \& Bottomore 1998, p.37). Marshall y Bottomore (1998) proponían tres dimensiones para la ciudadanía; la civil, la política y la social. Por ciudadanía civil, comprendían los derechos vinculados a las libertades de las personas. En cuanto a la dimensión política, la asociaban a la posibilidad de participar del ejercicio político. Por elemento social, entendían el bienestar económico y la seguridad social. Posterior al planteamiento clásico, la ciudadanía ha sido comprendida de distintas formas. Por ejemplo, desde el punto de vista de los derechos constitucionales, la ciudadanía es entendida como un conjunto de normas que regulan el estatus jurídico-político de los habitantes adscritos a un territorio determinado (Pérez 2004).

No obstante, las definiciones clásicas o jurídicas no permiten incorporar elementos sociales y subjetivos, necesarios para comprender cómo las personas se comportan en relación con el Estado. Para García (1995), la ciudadanía, más allá de los derechos reconocidos por el Estado en un territorio determinado, tiene que ver con las prácticas sociales y culturales que otorgan identidad y sentido de pertenencia a un grupo humano y que, en definitiva, les llevan a organizarse para la búsqueda colectiva del bienestar. Varias investigaciones (Pérez 2004; Heather 2007; Fleury 2007; Campero 2007; Gómez 2010) afirman que la expresión de la ciudadanía es inseparable de la forma de democracia con la que se asocia, y así como bajo un régimen autoritario no es posible su desarrollo, en una democracia de baja intensidad la participación ciudadanía también se reducirá a su mínima expresión, especialmente en su dimensión política (O’Donnell 1993). A partir de ello, Cortina (1997) propone elaborar una teoría de la ciudadanía, estrechamente vinculada a las teorías de democracia y justicia, ya que esto permitiría reforzar la democracia postliberal permitiendo la coexistencia de las demandas de justicia con las necesidades de identidad y pertenencia.

Respecto de la categoría “juventud”, es necesario aclarar que se asumirá la visión de que las y los jóvenes son actores/as sociales con identidades, heterogéneos/as, complejos/as y portadores/as de proyectos diferenciados en función de relaciones de clase, de género y etnia, y no solamente una categoría atravesada por asuntos etarios o generacionales (Duarte 2000; Reguillo 2000; Chaves 2010; Rodríguez 2015). La heterogeneidad de la juventud permite incorporar a la noción de ciudadanía derechos sociales y políticos emergentes, en tanto demandas, o que ya han sido conquistados por la suma de acciones en la vida cotidiana, incorporando como propias las demandas de otros actores que han ido ganando visibilidad, como personas homosexuales, pueblos originarios, feministas y grupos ecologistas, adicionando además temáticas también diversas como educación, igualdad de género, defensa de recursos naturales, derechos de grupos sociales históricamente excluidos, etc. Esta diversidad también se manifiesta en la diversificación de las acciones tradicionales de protesta, desde las más tradicionales, como realizar marchas, meetings, portar lienzos y lanzar panfletos, hasta acciones de carácter más carnavalescas y artísticas, como los flash mobbs, batucadas o performances (Fernández 2013).

Investigaciones respecto de las dinámicas de participación de los y las jóvenes a través de nuevas formas de organización, conocidas como "colectivos" permiten reconocer cómo estas experiencias giran en torno a propuestas sociales y culturales que muestran una funcionalidad que dista mucho de las dinámicas organizativas tradicionales. Estas nuevas formas de agrupación juvenil estarían caracterizadas por una organización no jerárquica, con autonomía de gestión y por el uso de acciones políticas directas y con 'consignas' colectivas más relacionadas con la vida cotidiana (Valenzuela 2007; Garcés 2010; Sandoval \& Hatibovic 2010). Para Valenzuela (2007) estas nuevas formas de relación juvenil con lo político, se caracterizarían por no estar 
orientadas a la conquista del poder a través del control del Estado, ya que se centran en temáticas más cercanas a la cotidianeidad, donde la política es vista como una posibilidad para la deconstrucción de un orden que se ha dado por natural, pero que es producto de la construcción hegemónica de quienes han dominado el discurso social. Se asume que esta tarea puede partir desde el nivel microsocial y permea a la sociedad en su conjunto (Aguirre \& Agustín 2015). La forma de su organización obedece a procesos de autogestión económica, ya que eso les da autonomía en sus decisiones, sus heterogéneas prácticas políticas se expresan a través múltiples formas como la danza, la música, el arte, los grafitis, entre otras, otorgándoles a sus expresiones organizativas un sello de pluralidad y democracia.

"La política en los jóvenes pasa por el deseo, la emotividad, la experiencia de un tiempo circular, el privilegio de los significantes sobre los significados... cuestiones que posibilitan ver a la política ya no como un sistema rígido de normas, sino como una red variable de creencias... Es una política con minúscula, que adquiere corporeidad en la práctica cotidiana de los actores, en los intersticios que los poderes no pueden vigilar" (Reguillo 2000, p.43).

En parte, estas nuevas configuraciones estarían dadas porque los/las jóvenes serían más sensibles que otros grupos etarios a los discursos y a las prácticas que abogan por la diversidad, la inclusión, el cuidado del medio ambiente y los derechos humanos y serían más proclives a luchar por las "pequeñas causas" (Garcés 2010).

Por otra parte, la incorporación de un enfoque de género, nos permite visibilizar procesos históricos, que a menudo son poco abordados disciplinariamente (Giordano 2012). Efectivamente, las especificidades de género en cuestiones relativas a la ciudadanía han sido relegadas, por varias razones. Simone de Beauvoir en el Segundo Beauvoir (1999), ya reconocía que las mujeres han sido solidarias de las causas "humanas", dejando de lado las reivindicaciones específicas de su género, ya que, de acuerdo con su análisis, las mujeres "viven dispersas entre los hombres, atadas por el medio ambiente, el trabajo, los intereses económicos, la condición social, a ciertos hombres -padre o marido- más estrechamente que a las demás mujeres" (Beauvoir 1999, p.21). Misma línea de razonamiento lleva a Pateman (1988) a pensar que habiendo un explícito contrato social, mucho más implícitamente mujeres y hombres han establecido un contrato sexual. A partir de ello, desde la teoría feminista se han analizando estas cuestiones, relacionándolas con las divisiones patriarcales clásicas de lo público y lo privado (Fernández 1994). En esta línea, Lister (2012) argumenta que la ciudadanía se encuentra absolutamente mediada por el género y a lo largo de su historia, la falsa idea de neutralidad de género, ha contribuido a invisibilizar la naturaleza de estas diferencias "La dicotomía público/privado y las cualidades masculina/femenina asociadas a ella están en el núcleo de la relación de ciudadanía generizada" (Lister 2012, p.80). Esta visión dicotómica pone a la política en el ámbito de lo público, dando con ello una pertenencia "natural" en ella a los hombres, quienes se encuentran simbólicamente asociados a este polo de la dicotomía (Fernández 1994). Secundariamente, lo privado queda despolitizado, relegado a las mujeres, los niños y niñas, y todos aquellos grupos de la población que no gozan de una ciudadanía plena, "las mujeres y los niños se incluyen en aquellas formas de sociabilidad correspondientes a su clase social, pero en tanto no son sujetos de contrato, su posibilidad de circulación se despliega en el mundo privado y no en el público, y no son ciudadanos en el pleno sentido sino que la figura jurídica en la que se inscriben es la de la tutela" (Fernández 1994, p.144). Esto ha supuesto grandes brechas en el mundo público, por ejemplo, específicamente en la representación política a nivel Latinoamericano (Martínez \& Garrido 2013; Ríos 2008), lo que permite, en el caso de las mujeres y otros grupos, hablar de ciudadanía 
2 De hecho, esta disyuntiva es la que lleva a popularizar el lema "Democracia en el país, en la casa y en la cama", propia de los movimientos de mujeres durante la dictadura chilena. restringida (Bareiro 2012). A esto, se suma la visión humanista clásica, que unifica lo humano en un ser masculino, blanco, clase media y heterosexual. La crítica a esta estructuración de la realidad simbólica es la que permitió a los movimientos feministas de los sesenta enarbolar el lema "lo personal es político" llamando con ello la atención sobre la necesidad de romper los límites entre lo público y lo privado o sobre la politicidad de lo personal (Acevedo 2012).

Es necesario en este punto, aunque de algún modo ya lo hemos hecho implícitamente, distinguir género de mujeres, ya que puede, desde un enfoque poco cuidadoso, homologarse ambos conceptos. Si bien en este artículo nos centramos en las miradas de las mujeres jóvenes, comprendemos el género como una construcción social relacional, no exclusiva de un grupo social, y adscribimos a la idea de que "género hace hincapié en todo un sistema de relaciones que puede incluir el sexo, pero que no está directamente determinado por éste ni tampoco es directamente determinante de la sexualidad" (Scott 1999, p.53). No obstante, es necesario reconocer también que el olvido histórico de los aspectos más o menos específicos que un sistema de sexo-género impone a ciertos cuerpos sexuados, en este caso, las mujeres, muestra la necesidad de un replanteamiento de una ciudadanía que incluya esos cuerpos; "La oposición binaria y el proceso social de las relaciones de género pasan a formar parte del significado del propio poder" (Scott 1999, p.73). Las mujeres no son género, pero mirar con género supone poder ver a las mujeres, también. Otro elemento a tener en cuenta es que el carácter de las formaciones de género particulares a una cultura e historia, se relaciona con las demandas que las mujeres hacen y los derechos que han ganado (Molyneux 2003), lo cual implica comprender que las jóvenes chilenas demandan, en tanto mujeres, asuntos diferentes o con diferentes énfasis que jóvenes mujeres de otros países latinoamericanos.

Kirkwood $(1985 ; 1986)$ relata cómo las causas políticas en Chile durante la dictadura son neutralizadas en relación al género, aun cuando la actuación de las mujeres en ese momento es fundamental. La autora muestra cómo se va pidiendo a las mujeres apoyar causas políticas y de clase, ya que "A la mujer no se la concibe sola" (Kirkwood 1985, p.64). Kirkwood advierte sobre el peligro de defender los sistemas políticos androcéntricos, invisibilizando las problemáticas de las mujeres chilenas en dictadura² ${ }^{2}$, situación que podría extrapolarse, a cuando en la actualidad se habla de "ciudadano" queriendo implicar la representatividad de este sujeto de todos y todas independiente de sus particularidades y requerimientos específicos. En Latinoamérica, en general, también se tensionó fuertemente la idea de ciudadanía con la ciudadanía de las mujeres durante las diversas dictaduras, donde dentro de la idea de violación a los derechos humanos no se incluía, por ejemplo, la falta de leyes estatales para lidiar contra la violencia contra las mujeres (Bareiro 2012). Así, se reconoce que uno de los grandes aportes de los movimientos feministas es la instalación de un discurso que incorpora una ciudadanía sexual, que desborda lo establecido en la noción clásica Marshalliana y que ha permitido, además, reivindicaciones de grupos LGTB (Bareiro 2012). En Latinoamérica la introducción de los asuntos de las mujeres en el Estado fue posible recién durante los procesos de restauración democrática (Brown 2014). En el caso particular de Chile, Valdés (2013), con una visión institucionalista, establece que desde el retorno a la democracia las mujeres han instalado progresivamente sus demandas en la agenda ciudadana, siendo la elección de Bachelet una muestra de ello. Valdés (2013) reconoce momentos históricos fundantes en esta progresión; la instalación de políticas de equidad, la instalación de políticas de igualdad y empoderamiento y, más contemporáneamente, una demanda por ciudadanía activa. Sin embargo, con los actuales debates instalados sobre aborto terapéutico, matrimonio igualitario y educación, ha quedado claro que existen varios temas pendientes en una agenda ciudadana para mujeres y jóvenes. 
Siguiendo la línea crítica de género, entonces, recientemente se han experimentado cambios en los enfoques sobre ciudadanía, yendo desde una concepción liberal hasta enfoques más contemporáneos, lo cual ha significado, de acuerdo a lo planteado por Zúñiga (2010),

"La inclusión del género en una doble vertiente: a) como factor explicativo de las asimetrías de poder traducidas en el goce diferenciado de derechos y b) como elemento normativo que se vertebra sobre una estrategia de igualación que tiene en cuenta la especificidad de género de los sujetos (entre otras diferencias)" (Zúñiga 2010, p.161).

Las mujeres jóvenes, de este modo, constituyen un grupo desde el cual podemos reconocer una forma de ciudadanía particular y emergente, imprescindible para entender la relación de la sociedad actual con la política.

\section{Metodología}

La investigación se ha desarrollado durante los años 2013-2014, y los resultados que se dan a conocer corresponden al análisis parcial de algunos de los datos obtenidos. El estudio se ha realizado desde un enfoque integrado (cualitativo-cuantitativo) utilizando la técnica CAWI (Computer Assisted WEB Interviewing). Se utilizaron cuestionarios diseñados para explorar la visión de las mujeres jóvenes respecto de la ciudadanía, tanto con preguntas abiertas como cerradas.

El análisis de la información correspondiente a las respuestas a las preguntas abiertas ha sido abordado con la utilización de técnicas de análisis de contenido, siguiendo un proceso de codificación abierta fundamentada en la determinación de densidad, para posteriormente proceder a la reducción analítica, en un proceso de contrastación constante con los referentes teóricos, lo cual fue asistido con el software de análisis de datos Atlas-ti 7.1, llevando a cabo en primer lugar un análisis lexicométrico con la herramienta Word Crunch de dicho software, lo que permitió visibilizar los conceptos con mayor frecuencia (densidad), para luego codificarlos, categorizarlos e interrelacionarlos.

Los resultados presentados corresponden al análisis de los datos de 156 cuestionarios respondidos online por mujeres jóvenes, de entre 18 y 29 años, de las cuales el $20 \%$ tenía entre 18 y 22 años, y el $80 \%$ de 23 a 29 años, y cuya procedencia era fundamentalmente de zonas urbanas de más 100 mil habitantes (75\%); mientras el $25 \%$ son de zonas urbanas de menos de 100 mil habitantes o de zonas rurales. El $85 \%$ poseía estudios de educación superior, en tanto que un $15 \%$ estaba aún cursando enseñanza media.

La difusión del instrumento y la convocatoria a responder se realizó mediante el uso de correos electrónicos y plataformas sociales como Twitter y Facebook, lo que puede estar introduciendo varios sesgos a la información recolectada. En primer lugar, porque quienes se sienten motivadas a responder una encuesta sobre ciudadanía probablemente tengan una actitud favorable a temáticas relativas a ésta, y segundo, porque son mujeres con acceso a las mencionadas redes sociales.

\section{Resultados}

\footnotetext{
${ }^{3}$ Ley $\mathrm{N}^{\mathrm{o}} 20$ 568, vigente desde el 31 de enero de 2012.
}

En primer lugar, es importante resaltar que el 75,7\% de las jóvenes que respondieron la encuesta, no se habían inscrito en los registros electorales, antes de la entrada en vigencia de la Ley de inscripción automática. ${ }^{3}$

Respecto de su apreciación global sobre el género y su relación con la ciudadanía, frente a la pregunta de si consideran que hombres y mujeres ejercen 
su ciudadanía de distintas formas, un $83 \%$ considera que sí, y solo un $13 \%$ cree que no hay diferencias.

Respecto de sus derechos en tanto ciudadanas, un $87 \%$ de las mujeres jóvenes considera que en Chile hombres y mujeres no gozan de los mismos derechos como ciudadanos/as, percibiendo inequidades y discriminaciones en distintos ámbitos que son considerados por las mujeres jóvenes como espacios o situaciones de discriminación de género, ordenados de mayor a menor, según densidad obtenida en el proceso de codificación, se obtienen los siguientes ámbitos de discriminación por género:

1. Situaciones laborales.

2. Violencia patriarcal en situaciones públicas (calle y situaciones sociales).

3. Roles y estereotipos impuestos a las mujeres.

4. Salud y previsión.

5. Sexualidad y reproducción.

Es necesario destacar que los ámbitos precedentes, como se verá en el análisis detallado de cada uno de ellos, están directamente asociados entre sí, siendo a veces inseparables, en ocasiones estando algunos a la base de los otros. Por ejemplo, las posibilidades de actuar con autodeterminación, se encuentran condicionadas a la consecución previa de los derechos políticos.

El análisis de la información obtenida, segregada por respuestas nos permite distinguir las situaciones percibidas como ámbitos o espacios de mayor discriminación por parte de las mujeres jóvenes, donde destacan las situaciones vinculadas al trabajo y, en general, a los ámbitos laborales, aparecen como los espacios de mayor discriminación, algunos de ellos derivadas de la doble condición de jóvenes y de mujeres. Las particularidades de este ámbito están dadas por diferencias en las rentas obtenidas por igual función desempeñada, discriminación respecto de posibilidades de contratación, conservación del trabajo una vez obtenido, acoso laboral y diferencias respecto de las posibilidades de ascenso. Lo anterior, puede ser ilustrado con los siguientes extractos de respuestas:

"A la hora de buscar empleo, una por ser mujer, la otra por ser joven"

"En el ámbito laboral, las bromas de parte de los hombres, y por ser mujer tengo que demostrar mayor capacidad para sentirme valorada"

"Ganar lo mismo que los hombres, terminar con los altos costos de planes de salud, en definitiva reflejar verdadera y objetivamente una igualdad de condiciones con el hombre, eso va desde los sueldos, hasta el post natal".

"Igualdad de derechos centrado en: Sueldo igualitario para hombres y mujeres, obtención de cargos altos independientes del género, igualdad en derechos de Isapres, cumplimiento de pre y post natal en empresas públicas o privadas, con contrato o a honorarios, jubilación digna y acorde al sueldo que se obtuvo, de por vida".

"La igualdad de sueldo con respecto al trabajo que un hombre realiza, la misma actividad laboral y que no sea discriminada por edad o sólo por ser mujer".

En segundo lugar, se señala como ámbito de discriminación el acoso y la violencia patriarcal (verbal, simbólica e incluso física) en situaciones públicas y en situaciones sociales. Específicamente, la calle es percibida como un espacio que excluye a las mujeres, que las obliga a vestirse o a comportarse de determinado modo, y las expone a ser el objeto de burlas o acoso callejero (en 
Chile, naturalizado bajo la denominación de "piropos”) en alusión a su cuerpo o su forma de vestir.

"En la calle, constantes "piropos", chistes me hacen sentir que la calle no es para que yo ande en ella. No es mi lugar"

"En la calle, todos los días... los hombres se sienten con el derecho de ofenderte y propasarse como si fuéramos un objeto más que decora el medio ambiente”

"Al manejar, siento que me respetan menos, o esperan a que me equivoque y si lo hago, me gritan $<<$ tenía que ser mujer $>>$ "

"Se requieren leyes más severas ante la violencia de género, mayor protección a las víctimas, y mayor fiscalización del cumplimiento de estas leyes".

Un tercer ámbito de discriminación se asocia a lo que las jóvenes consideran como roles y estereotipos impuestos socialmente a las mujeres, y que se encuentran vinculados a labores de cuidado tradicionalmente asociadas a la dicotomía público privado, que asigna principalmente a las mujeres el deber del cuidado de los hijos e hijas, la realización de las labores domésticas y el deber de estar siempre "presentables". La asociación de este ámbito a los derechos ciudadanos da cuenta de procesos de generización de la ciudadanía y de la incorporación de ámbitos no tradicionales que ponen en tela de juicio la separación público/privado.

"Desde tener que comportarme de cierta manera, vestirme de cierta forma, hasta la misma depilación"

"Cuando se me cataloga como principal responsable de la crianza de los niños, cuando se me valora como mujer sólo en tanto mi capacidad reproductiva"

En cuarto lugar, y muy ligado al ámbito laboral, se encuentra la percepción de discriminación respecto de el acceso igualitario a la Salud Previsional. Esto en Chile puede estar acentuado por la aplicación extrema de las ideas neoliberales, que transformaron el derecho a la salud en un bien de consumo, al que se tiene acceso, no como una garantía universal, si no como un servicio particular al que se accede según la capacidad de pago y los factores de riesgo asociados a las personas. En el caso de las mujeres jóvenes, las instituciones privadas de salud consideran que las posibilidades de embarazo constituyen un factor de riesgo, por lo que deben pagar más por obtener seguros de salud similares en cobertura, a los obtenidos por los hombres.

\section{"Planes de cotización previsional por debajo de los hombres" \\ "Los descuentos por salud en Isapres por ser mujer en edad fértil son un escándalo" \\ "En cuanto a los planes de salud por ejemplo si se es mujer y se está en edad reproductiva todo es de mayor costo"}

En quinto lugar, y también asociado a ámbitos analizados precedentemente, como las discriminaciones asociadas a mandatos sociales, o al acceso a la salud, se relevan los temas vinculados a la Sexualidad y la Reproducción. Aquí las demandas por equidad asocian directamente lo reproductivo con lo político.

"Que siempre se me vea como puta por ejercer mi sexualidad libremente, en no poder decidir libremente sobre en mi cuerpo, en mi decisión de no ser madre"

"Cuando se me considera como un cuerpo para otro e incapaz de tomar decisiones sobre mi propio cuerpo"

"Cuando estaba embarazada me despidieron y la razón fue porque estaba embarazada y la empresa no tenia plata para mantener a mujeres embarazadas"

Consecuente con los ámbitos percibidos como espacios de discriminación, los temas que las mujeres jóvenes consideran como necesarios de incorporar en 
una agenda ciudadana, son: Derechos Sexuales y Reproductivos; Equidad Laboral; Equidad Salarial; Educación; Violencia Patriarcal; Participación Política, Derechos Sociales, y Previsión Social. Esto, además de ser consistente con los ámbitos mencionados como problemáticos, permite afirmar que las mujeres jóvenes tienen una visión amplia de la ciudadanía, que politiza temas que habitualmente son atribuidos al ámbito de lo privado, rompiendo el límite que desde la concepción clásica de la ciudadanía se establece entre lo público y privado. De esta forma, temas como "el cuidado", "La reproducción” y "el aborto", aparecen como temas que se exige sean asumidos desde el ámbito de la relación del Estado con la sociedad civil.

La siguiente red conceptual (Gráfico 1), muestra la relación que se establece entre los ámbitos que las mujeres jóvenes reclaman como ciudadanas, quedando en evidencia la amplitud y las asociaciones. La primera cifra en el paréntesis muestra la densidad que da fundamento a la generación de la categoría, en tanto que el segundo número da cuenta de la cantidad de asociaciones establecidas entre las categorías:

Las conexiones muestran las asociaciones existentes entre las distintas categorías, las que han sido agrupadas bajo la categoría más global denominada "Derechos como ciudadanas". Allí se puede apreciar que la categoría de mayor densidad es la de Igualdad de Derechos, con 77 menciones, seguido de Derechos Sexuales y Reproductivos, con 74. En tanto que la que concentra mayor número de relaciones, es la de Derechos Sociales, con 5 asociaciones.

Es interesante ver que los derechos sociales son vistos como compuestos por previsión, salud, educación y protección, ámbitos recientemente relevados en los últimos movimientos sociales chilenos, especialmente en el año 2011, lo cual coincide con que además, la más densa de todas resulta ser educación. De este mismo modo, la igualdad de derechos es fuertemente asociada con los derechos laborales, sociales y sexuales y reproductivos, siendo estos últimos ligeramente más densos que los otros dos. Esto se conecta con la perspectiva de

Gráfico 1 - Ámbitos y relaciones de ciudadanía

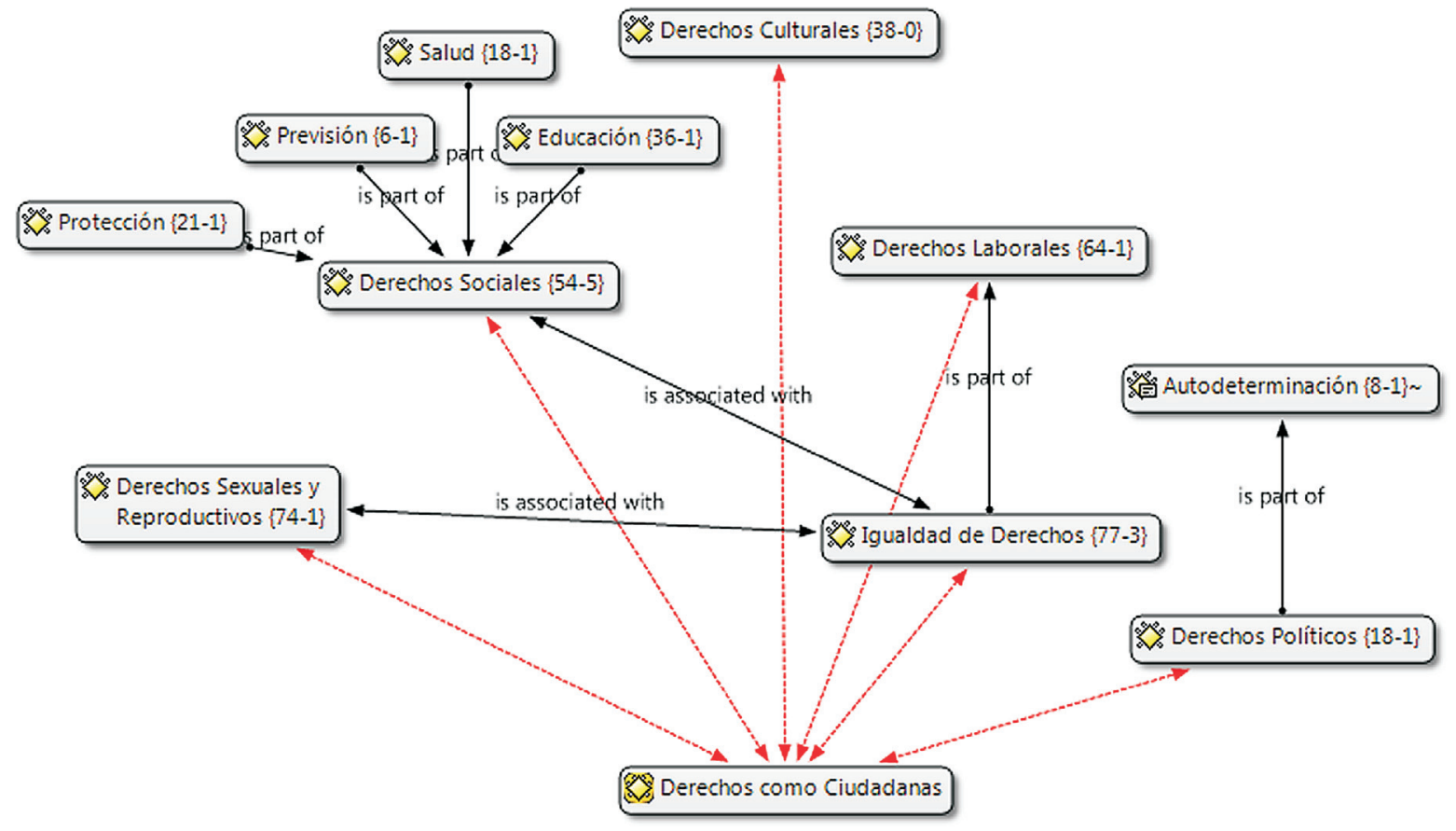

Fuente: elaboración propia. 
género, puesto que entendemos que aquello en lo que las mujeres acusan mayor urgencia de igualdad son aquellos temas en los cuales se ven también más directamente afectadas, en tanto grupo. Coincidentemente, temas como aborto y libre determinación sobre el cuerpo son asuntos recurrentes en las respuestas.

Llama la atención que la categoría de derechos políticos no se encuentre fuertemente asociada a las otras, lo cual, a nuestro juicio, denota que la ciudadanía no es vista como una dimensión solamente política, teniendo los derechos sociales un mismo nivel jerárquico de relación con la ciudadanía.

\section{Conclusiones}

En primer lugar, es posible afirmar que se evidencian particularidades vinculadas a la condición de género y que configuran dimensiones distintivas en torno a la representación y práctica de la ciudadanía, la cual es asumida de forma directamente asociada a la condición de mujeres, por las jóvenes, por lo que podríamos concordar con lo planteado por Lister (2012), quien sostiene que existe histórica y actualmente una generización de la ciudadanía. La fuerte discriminación de género que las mujeres jóvenes perciben respecto de los temas ciudadanos actuales evidencian que la tensión entre la ciudadanía y la ciudadanía de las mujeres (Bareiro 2012) aún persiste.

De esta especificidad emergen categorías como: la sexualidad, el cuerpo, la reproducción, lo laboral, la discriminación y la violencia, las que aparecen fuertemente asociadas a lo político e institucional, lo cual pone en tensión las categorías clásicas asociadas a la ciudadanía, y ratifica lo planteado por Reguillo (2000) en cuanto a que la juventud es una categoría atravesada por relaciones de género, y que la política en estos grupos etarios se centra en aspectos micro-sociales y cotidianos. Es necesario, sin embargo, pensar en la posibilidad de que estos, aparentemente "pequeños" temas, sean tal vez los grandes temas políticos con los que deberá lidiar la investigación sobre ciudadanía y política. Es decir, que esta no sea una forma peculiar de asumir lo político desde la juventud, sino que se esté configurando una nueva manera de ciudadanía, con nuevas dimensiones que llegaron para quedarse, y que imponen a los estados contemporáneos desafíos mucho más complejos. Esto pone de manifiesta la posibilidad de que estemos frente al surgimiento de un sujeto político imprevisto (Acevedo 2012), que además de percibir la precariedad general de la vida cotidiana, sea capaz de movilizarse a partir de la precariedad cotidiana específica de las mujeres y otras causas ligadas a subjetividades precarias (Fernández 1994).

Las mujeres jóvenes participantes en este estudio se reconocen como ciudadanas sexuales, en un Estado en el cual son, a la vez que sujetas privadas, objetos públicos, ya que "el cuerpo de las mujeres con su capacidad reproductiva continúa siendo el mayor terreno de disputa legal, el más normado y en búsqueda de su autonomía" (Bareiro 2012, p.45). La imagen de un cuerpo político en un espacio público, tiene su expresión más concreta en el acoso callejero, la percepción de la calle y la ciudad como espacios de violencia e inadecuación y el establecimiento de la topografía del miedo para las mujeres, limitando su derecho a circular y actuar en este espacio (Falú 2012).

Llama fuertemente la atención el nivel de elaboración crítica que presentan las respuestas de las encuestadas sobre los asuntos de género, especialmente respecto a sus experiencias de primera mano. Esto que a primera vista se contradice un poco con resultados de investigaciones hechas por nuestro grupo con otros tipo de poblaciones de mujeres, que tienden a demostrar un nivel mayor de conformidad y normalización de las cuestiones de género. Estas discrepancias pueden ser explicadas con el sesgo muestral, ya que primero, la 
mayoría de quienes han contestado el cuestionario poseen estudios universitarios, y en segundo lugar, con el sesgo que se explicitara en el apartado metodológico respecto a las características pro-ciudadanía o de mayor politización de quienes tienden a responder a las convocatorias para participar voluntariamente en este tipo de estudios.

Como conclusión general, se puede citar de la necesidad de que las instituciones democráticas reencanten a las personas jóvenes, incorporando nuevas dimensiones como la de género, cultura, ecología, como parte del diseño de políticas públicas, como en la necesidad de respetar las acciones de carácter autónomo y las nuevas configuraciones de la acción política, que desde las propias iniciativas buscan fortalecer la capacidad agéntica de las mujeres jóvenes y otros grupos.

Como proyecciones de este estudio se espera en próximas investigaciones profundizar desde una perspectiva cualitativa respecto de las representaciones sociales que los/las jóvenes poseen sobre ciudadanía, desde una perspectiva de género. Además, se considera necesario profundizar respecto de las nuevas prácticas ciudadanas de las jóvenes, las nuevas formas de participación ciudadana que se alejan de los partidos políticos tradicionales, y que optan por formas de acción directa o por medio de la conformación de Colectivos que trascienden la esfera política tradicional y asumen temas emergentes como los derechos de los animales, la preservación del medio ambiente o los derechos de las "minorías".

También se considera conveniente analizar cómo, la transición de un proceso de socialización política de las mujeres, que históricamente se ha realizado siguiendo modelos masculinizados y en el cause de las organizaciones tradicionales, hacia un proceso de socialización que en la actualidad está más mediada por la influencia de pares, los movimientos feministas o por la participación en organizaciones no convencionales como los colectivos, están configurando nuevas aproximaciones, y dimensiones de la ciudadanía.

Bruno Bivort (bbivort@ubiobio.cl) es Magister en Investigación Social y Desarrollo por la Universidad de Concepción (Chile), investigador del Grupo de Investigación GECIEQ, y académico adscrito al Departamento de Ciencias Sociales de la Universidad del Bío-Bío, Chillán, Chile. Vínculo institucional: Grupo de Investigación GECIEQ, Departamento de Ciencias Sociales, Universidad del Bío-Bío, Chillán, Chile.

Soledad Martínez-Labrín (cmartine@ubiobio.cl) es Magister en Género y Sociedad por la Universidad de Southampton (Inglaterra), investigadora del Grupo de Investigación GECIEQ, y académica adscrita al Departamento de Ciencias Sociales de la Universidad del Bío-Bío, Chillán, Chile. Vínculo institucional: Grupo de Investigación GECIEQ, Departamento de Ciencias Sociales, Universidad del Bío-Bío, Chillán, Chile.

Cristian Orellana (corellana@ubiobio.cl) es Magister en Investigación Social y Desarrollo por la Universidad de Concepción (Chile), investigador del Grupo de Investigación GECIEQ, y académico adscrito al Departamento de Ciencias Sociales de la Universidad del Bío-Bío, Chillán, Chile. Vínculo institucional: Grupo de Investigación GECIEQ, Departamento de Ciencias Sociales, Universidad del Bío-Bío, Chillán, Chile.

Fernando Farías (ffarias@ubiobio.cl) es Magister en Investigación Social y Desarrollo por la Universidad de Concepción (Chile), investigador del Grupo de Investigación GECIEQ, y académico adscrito al Departamento de Ciencias Sociales de la Universidad del Bío-Bío, Chillán, Chile. Vínculo institucional: Grupo de Investigación GECIEQ, Departamento de Ciencias Sociales, Universidad del Bío-Bío, Chillán, Chile.

\section{Referencias}

Acevedo, M., 2012. Feminismo: lo que desató la politicidad de lo personal. Em Equipo latinoamericano de Justicia y Género. Autonomía y feminismo Siglo XXI: Escritos en homenaje a Haydée Birgin. Buenos Aires: Biblos.

Aguirre, F. \& Agustín, O., 2015. Más allá del malestar. Una hipótesis sociológica sobre el significado político del movimiento estudiantil chileno. Revista de Sociologia e Política, 23(53), pp.147-162. DOI: 10.1590/1678-987315235308 
Aquin, N., 2003. El trabajo social comunitario en las actuales condiciones: fortalecer la ciudadanía. In N. Aquín, ed. Ensayos sobre ciudadanía, reflexiones desde el trabajo social. Buenos Aires: Espacio.

Bareiro, L., 2012. Ciudadanas en Estados mutantes. In Equipo latinoamericano de Justicia y Género. Autonomía y feminismo Siglo XXI: Escritos en homenaje a Haydée Birgin. Buenos Aires: Biblos.

Bermúdez, N.; Savino, L. \& Zenklussen, L., 2004. Representaciones sobre democracia y participación en la juventud de la ciudad de Córdoba. Cuadernos de la Facultad de Humanidades y Ciencias Sociales, 22, pp.122-150.

Bonvillani, A.; Palermo, A.; Vázquez, M. \& Vommaro, P., 2008. Juventud y política en la Argentina (1968-2008): Hacia la construcción de un estado del arte. Revista Argentina de Sociología, 6(11), pp.44-73.

Brown, J., 2014. Mujeres y Ciudadanía en Argentina. Debates teóricos y políticos sobre derechos (no)reproductivos y sexuales (1990-2006). Buenos Aires: Teseo.

Campero, G., 2007. Trabajo y Ciudadanía. In F. Calderón, ed. Ciudadanía y desarrollo humano. Buenos Aires: Siglo Veintiuno.

Chaves, M., 2010. Jóvenes, Territorios y Complicidades: Una antropología de la juventud urbana. Buenos Aires: Espacio.

Cortina, A., 1997. Ciudadanos del mundo, hacia una teoría de la ciudadanía. Madrid: Alianza.

Beauvoir, S., 1999. El segundo sexo. Buenos Aires: Editorial Sudamericana.

Duarte, K., 2000. ¿Juventud o Juventudes?: Acerca de cómo mirar y remirar a las juventudes de nuestro continente. Última Década, 8(13), pp.59-77. DOI: 10.4067/s0718-22362000000200004

Falú, A., 2012. Profundizando en los derechos y en las autonomías: las mujeres en las ciudades. In Equipo latinoamericano de Justicia y Género. Autonomía y feminismo Siglo XXI: Escritos en homenaje a Haydée Birgin. Buenos Aires: Biblos.

Fernández, A., 1994. La Mujer de la ilusión. Pactos y contratos entre hombres y mujeres. Buenos Aires: Paidos. , 2013. El espacio público en disputa: Manifestaciones políticas, ciudad y ciudadanía en el Chile actual. Psicoperspectivas, 12(2), pp.28-37.

Fleury, S., 2007. Los patrones de exclusión e inclusión social. In F. Calderón, ed. Ciudadanía y desarrollo humano. Buenos Aires: Siglo Veintiuno.

Garcés, A., 2010. De organizaciones a colectivos juveniles: Panorama de la participación política juvenil. Última Década, 18(32), pp.61-83. DOI: 10.4067/s0718-22362010000100004

García, N., 1995. Consumidores y Ciudadanos. Conflictos multiculturales de la globalización. México: Grijalbo.

Giordano, V., 2012. Ciudadanas Incapaces. La construcción de los derechos civiles de las mujeres en Argentina, Brasil, Chile y Uruguay en el siglo XX. Bueno Aires: Teseo.

Gómez, J., 2010. Política, democracia y ciudadanía en una sociedad neoliberal (1990- 2010). Santiago: Arcis.

Gutiérrez, S., 2011. Representaciones sociales y construcción de la ciudadanía en jóvenes universitarios. Sinéctica, 36, pp.1-18.

Heather, D., 2007. Ciudadanía, una breve historia. Madrid: Alianza.

Kirkwood, J., 1985. Feministas y Políticas. Nueva Sociedad, 78, pp.62-70. 1996. Ser Política en Chile. Las feministas y los partidos. Santiago de Chile: Cuarto Propio.

Lister, R., 2012. Ciudadanía y Género. Debate Feminista, 23(45), pp.79-94.

Marshall, T. \& Bottomore, T., 1998. Ciudadanía y clase social. Buenos Aires: Losada.

Martínez, M. \& Garrido, A. 2013. Representación de género y calidad de la democracia: la doble brecha en América Latina. Revista Debates, 7(1), pp.151-173.

O’Donnell, G., 1993. Estado, Democratización y Ciudadanía. Nueva Sociedad, 128, pp.62-87.

Molyneux, M., 2003. Women's Movements in International Perspective: Latin America and Beyond. London: University of London.

Paredes, J., 2011. Ciudadanía, Participación y Democracia Deuda y Déficit en los 20 años de "Democracia" en Chile. Polis, 10(28), pp.473-499. DOI: 10.4067/s0718-65682011000100022

Pateman, C., 1988. The Sexual Contract. Stanford: Stanford University Press.

Pérez, A., 2004. ¿Ciberciudadani@o ciudadani@.com? Barcelona: Gedisa.

Reguillo, R., 2000. Emergencia de culturas juveniles. Estrategias del desencanto. Buenos Aires: Norma.

Ríos, M., 2008. Introducción. In M. Ríos, ed. Mujer y Política: el impacto de las cuotas de género en América Latina. Santiago de Chile: FLACSO.

Rodríguez, E., 2010. Políticas públicas de juventud en América Latina: Avances concretados y desafíos a encarar en el Marco del Año Internacional de la Juventud. Santiago de Chile: UNESCO.

2015. A modo de prólogo: Estudios sobre juventudes en América Latina: Un mosaico de realidades diversas pero convergentes, a caracterizar más y mejor. In H. Cubides; S. Borelli; R. Unda \& M. Vásquez, eds. Juventudes Latinoamericanas. Prácticas socioculturales, políticas y políticas públicas. Buenos Aires: CLACSO.

Sandoval, J.M., 2012. La desconfianza de los jóvenes: sustrato del malestar social. Última Década, 20(36), pp.43-70. DOI: $10.4067 / \mathrm{s} 0718-22362012000100003$

Sandoval, J.M. \& Hatibovic, F., 2010. Socialización política y juventud: el caso de las trayectorias ciudadanas de los estudiantes universitarios de la región de Valparaíso. Última Década, 18(32), pp.11-36. DOI: 10.4067/s071822362010000100002

Scott, J., 1999. Género e Historia. Ciudad de México: Fondo de Cultura Económica.

Toro, S., 2008. De lo épico a lo cotidiano: Jóvenes y generaciones políticas en Chile. Revista de Ciencia Política, 28(2), pp.143-160. DOI: $10.4067 /$ s0718-090x2008000200006 
Valenzuela, K., 2007. Colectivos juveniles: ¿inmadurez política o afirmación de otras políticas posibles? Última Década, 15(26), pp.31-52. DOI: 10.4067/s0718-22362007000100003

Valdés, T., 2013. Acción Política de Mujeres 1990-2006: Institucionalizando la equidad de género. In C. Mora, ed. Desigualdad en Chile: La Continua Relevancia del Género. Santiago de Chile: Universidad Alberto Hurtado.

Zarzuri, R., 2010. Tensiones y desafíos en la participación política juvenil en Chile. Utopía y Praxis Latinoamericana, 15(50), pp.103-115.

Zúñiga, Y., 2010 Ciudadanía y Género: Representaciones Y Conceptualizaciones en el Pensamiento Moderno y Contemporáneo. Revista de Derecho, 17(2), pp.133-163. DOI: 10.4067/s0718-97532010000200006

Young women and citizenship in Chile: a look onto new political configurations

\begin{abstract}
In the context of changes in the ways in which young people are linked with the State as citizens, this paper aims to identify and analyze the distinctive dimensions that could provide greater depth and extent to the category of Citizenship, from the perspective of young women, making visible the particularities associated to their status as youth and women. Using an integrated methodology, this exploratory study carries out the processing of the answers of 156 young women to an on-line mixed questionnaire about citizenship and gender. The vision of rights prioritized by women as citizens is analyzed, the specific issues they consider necessary to push from a citizens' agenda and special emphasis is placed on situations or areas perceived as spaces of gender discrimination by women, which are usually unnoticed in citizenship studies, which do not incorporate the gender dimension in their analysis. The later allows us to give account for a greater conceptual complexity, to look at citizenship beyond traditional conceptions.
\end{abstract}

KEYWORDS: gender; citizenship; young women; discrimination; political participation.

This is an Open Access article distributed under the terms of the Creative Commons Attribution Non-Commercial License which permits unrestricted non-commercial use, distribution, and reproduction in any medium provided the original work is properly cited. 